\title{
Recueil des pièces qui ont remporté le prix de l'Académie Royale des Sciences depuis 1720 jusqu'en 1772
}

Par Alvin E. Jaeggli

Avant-propos

La Bibliothèque de l'Ecole Polytechnique de Zurich a récemment pu acquérir les quatre premiers volumes du Recueil des pièces qui ont remporté le prix de l'Académie Royale des Sciences depuis leur fondation..., volumes, qui, déjà en 1777, lors de l'apparition du neuvième et dernier volume de cette série, ont été déclarés trèsrares par l'éditeur.

En catalogant cette collection qui sans doute est assez curieux pour l'histoire des sciences, j'ai cru qu'il serait de quelque utilité pour les chercheurs comme pour nos propres travaux, de rassembler les titres des pièces par ordre alphabétique des auteurs en ajoutant des indications bio-bibliographiques. Pour en donner une liste complète, j'y ai inclus les pièces qui se trouvent dans les tomes V-IX, indications que j'ai tirées d'un exemplaire de la Bibliothèque Centrale de Zurich. Il me semble remarquable que parmi les 65 pièces, dont nous connaissons l'auteur, 27 sont rédigées par des Suisses. Les Italiens ont contribué quatre titres, les Allemands un seul. Je ne pense pas de loin que ce fait énonce une espèce de rang national dans les sciences. Mais n'oublions pas que, lorsque le Journal des Sçavans, première revue scientifique en Europe, venait de paraître en 1665, les Allemands s'étaient scandalisés du fait qu'un tel organe osait se défaire du Latin.

Ce petit catalogue n'étant qu'un fruit de travail occasionnel ne peut s'occuper de la révélation des Anonymes que j'ai ajouté à la fin de la liste des auteurs. Deux exceptions ont été faites: Le numéro 30 dont l'auteur a été poliment présenté comme «jeune Dame d'un haut rang», et le numéro 65 qui était rédigé par «un de nos premiers poètes », n'étaient pas difficiles à révéler. Madame du Chastelet qui avait le plaisir de remporter le prix de l'Académie en même temps que son ami Voltaire, est assez connue par sa belle traduction des Philosophiae naturalis principia mathematica, et Voltaire qui aussi s'intéressait fort à la philosophie de Newton, a présenté en 1739 un mémoire à l'Académie de Bologne, sur un ouvrage de physique de la marquise. Il est certain que les deux pièces, couronnées à la même année par l'Académie Royale et portant presque le même titre, étaient le fruit d'une conversation scientifique entre Madame du Chastelet et Voltaire.

L'Académie des Sciences n'a pas, dès le moment de sa fondation, envisagé un Journal pour divulguer au monde des résultats obtenus, comme le pratiquait 
la Royal Society de Londres avec ses Philosophical Transactions. Pourtant de grands ouvrages comme des opuscules qui étaient les fruits des travaux des membres de l'Académie paraissent chez les libraires parisiens bientôt, sans titre commun et périodique. Pour les petits ouvrages, c'était le Journal des Sçavans, une revue privée datant de 1665, qui servait de point de ralliement. En 1730 seulement, Bernard de Fontenelle, alors Secrétaire de l'Académie, commençait à recueillir les articles des Académiciens, parus premièrement dans le Journal des Sçavans. C'est dans le tome X de son Histoire de l'Académie Royale des Sciences de 1666 à 1699 qu'on les retrouve.

En 1691, une première publication mensuelle fut annoncée dans le Journal des Sçavans sous le titre Mémoires de mathématique et de physique tiréz des registres de l'Académie des Sciences. Cette publication tenait un an et demi, puis elle s'arrêta. La prochaine publication rétrospective et historique fut rédigé par J. B. Du Hamel sous le titre Regiae Scientiarum Academiae Historia, Paris 1698, chez Etienne Michallet. Il y résumait l'essentiel des Registres dès 1666 jusqu'en 1696. En 1699, l'Abbé Bignon présentait un nouvel Règlement pour l'Académie qui fut admis. Deux propositions nous y intéressent le plus : la publication dans chaque année des Mémoires et l'examen des machines et des découvertes nouvelles faites par le public, ce qui présentait l'idée d'un «Patentamt». Dès lors l'Académie avait son porte-voix régulier qui fut publié successivement sous les titres suivants :

Histoire de l'Académie Royale des Sciences depuis 1699 jusqu'à 1790, avec les Mémoires de Mathématique et de Physique...

Paris $1702-1797$

en 92 volumes

Recueil des Pièces qui ont remporté le prix de l'Académie Royale des Sciences depuis 1720 jusqu'en 1772.

Paris $1721-1777$

en 9 volumes

Mémoires de l'Académie Royale des Sciences depuis 1666 jusqu'à 1699.

Paris 1729-1732

en 9 volumes

Machines et Inventions approuvées par l'Académie... éd. Jean-Gaffin Gallon.

Paris $1735-1777$

en 7 volumes

Mémoires de l'Institut National des Sciences et Arts. Sciences Mathématiques et Physiques (an IV-1805).

Paris an VI (1798)-1806

en 6 volumes

Mémoires présentés à l'Institut des Sciences, Lettres et Arts par divers savans et lus dans ses assemblées. Sciences mathématiques et physiques.

Paris 1805-1811

en 2 volumes

Procès-verbaux des Séances d'Académie tenues depuis la fondation de l'Institut, jusqu'au mois d'août 1835.

Hendaye 1910-1922

en 10 volumes 
Comptes rendus hebdomadaires des Séances de l'Académie des Sciences à Paris.

Paris 1835-1965

en 261 volumes

Depuis 1966 (volume 262) les Comptes rendus sont divisés en séries qui portent les chiffres A/B, C, D.

1. Bernoulli, Daniel I (1700-1782). Discours sur la manière la plus parfaite de conserver sur mer l'égalité du mouvement des Clepsidres, sabliers. 1725. 24 p., 1 pl.

2. - Recherches physiques et astronomiques sur le problème proposé pour la seconde fois par l'Académie...[De l'inclination mutuelle des planètes]. 1734. p. 93-144.

T. III

3. - Réflexions sur la meilleure figure à donner aux ancres, et la meilleure manière de les essayer. 1737. p. 47-84, $1 \mathrm{pl}$.

T. III

4. - Traité sur le flux et reflux de la mer. 1740. p. 53-191, 3 pl.

T. IV

5. - Mémoire sur la manière de construire les boussoles d'inclinaison, pour faire avec le plus de précision qu'il est possible, les observations de l'inclinaison de l'aiguille aimantée, tant sur mer que sur terre. 1743. p.1-61, 1 pl.

T. V

6. - Recherches méchaniques et astronomiques, sur la question: La meilleure manière de trouver l'heure en mer, par observation. 1745. p.1-110, 3 pl.

7. - Sur la nature et la cause des courans, et la meilleure manière de les déterminer. 1749 et 1751. p. $1-108,1 \mathrm{pl}$.

T. VI

T. VII

8. - Recherches sur la manière la plus avantageuse de suppléer à l'action du vent sur les grands vaisseaux, soit en y appliquant les rames, soit en y employant quelqu'autre moyen que ce puisse être. Fondées sur une nouvelle théorie de l'économie des forces et des effets. 1753. p. 1-99, 1 pl.

T. VII

9. - Mémoire sur la manière la plus avantageuse de suppléer à l'action du vent sur les grandes vaisseaux. [En latin.] 1753. p. 1-47, 2 pl.

T. VIII

10. - Principes hydrostatiques et méchaniques, sur la question proposée: Quelle est la meilleure manière de diminuer le roulis et le tangage d'un navire. 1757. p. 1-96, 1 pl.

T. VIII

11. - et Johann Bernoulli. Nouveaux principes de méchanique et de physique, tendans à expliquer la nature et les propriétés de l'aiman. 1746. p.115-144, 1 pl.

T. V

12. Bernoulli, Johann I (1667-1748). Discours sur les loix de la communication du mouvement. 1724 et $1726.110 \mathrm{p} ., 5 \mathrm{pl}$.

T. I

13. - Nouvelles pensées sur le système de Descartes, et sur la manière d'en déduire les orbites et les aphélies des planètes. 1730. 44 p., 1 pl.

T. II

14. - Essai d'une nouvelle physique céleste, servant à expliquer les principaux phénomènes du ciel, et en particulier la cause physique de l'inclinaison des orbites des planètes par rapport au plan de l'équateur du soleil. 1734.91 p., $1 \mathrm{pl}$. 
15. Bernoulli, Johann II (1710-1790). Recherches physiques et géométriques sur la question: Comment se fait la propagation de la lumière. $1736.66 \mathrm{p}$., 1 pl.

16. - Discours sur les ancres. 1737. 32 p., 3 pl.

T. III

17. - Discours sur le cabestan. 1741. p. 1-28, 3 pl.

T. V

18. Bilfinger, Georg Bernhard (1693-1750). De causa gravitatis physica generali disquisitio experimentalis. $1728.40 \mathrm{p} ., 2 \mathrm{pl}$.

T. II

19. Bosc d'Antic, Paul (1726-1784). Mémoire sur le prix proposé : Quels sont les moyens les plus propres à porter l'économie et la perfection dans les verreries de France? 1760. p. 1-53, 2 pl.

20. Boscovich, Ruggiero Giuseppe (1711-1787). [De inequalitatibus quas Saturnus et Jupiter sibi mutuo videntur inducere. 1752.]

- Prévu pour l'impression par l'Académie, mais publié à Rome en 1756.

21. Bossut, Charles (1730-1814). Mémoire sur l'arrimage des navires. 1761. p. $1-76,3$ pl.

T. VII

22. - Recherches sur les altérations que la résistance de l'éther peut produire dans le mouvement moyen des planètes. 1762. p.1-67, 1 pl.

23. Bouguer, Pierre (1698-1758). De la mâture des vaisseaux. 1727. 164 p., 5 pl.

T. VIII

T. I

24. - De la méthode d'observer exactement sur mer la hauteur des astres. 1729. 72 p., 2 pl.

T. II

25. - De la méthode d'observer en mer la déclinaison de la boussole. 1731. $67 \mathrm{p}$., 2 pl.

T. II

26. - Entretiens sur la cause de l'inclinaison des orbites des planètes. 1732 et 1734. 63 p., 2 pl.

T. II

27. Bourdé de Villehuet, Jacques (1730-1789). De l'arrimage du navire. 1766. p. 1-30.

T. IX

Bulfinger, G. B., voir Bilfinger, Georg Bernhard

28. Camus, Charles Etienne Louis (1699-1768). De la mâture des vaisseaux. 1727. 65 p., 3 pl.

T. II

29. Cavalleri, Antoine (1698-1763). Dissertation sur la cause physique du flux et du reflux de la mer. 1740. 51 p., 1 pl.

T. IV

30. Chastelet, Gabrielle Emilie Le Tonnelier de Breteuil, Mqse du (1706-1749). Dissertation sur la nature et la propagation du feu. 1738. p. 81-168. [Anonyme]

31. Créqui, Louis-Marie de (1705-1741). Explication de la nature du feu et de sa propagation. 1738. p. 55-80.

T. IV

32. Crousaz, Jean Pierre de (1663-1750). Discours sur le principe, la nature, et la communication du mouvement. $1720.67 \mathrm{p}$.

T. I

33. Delorme (de l'Académie de Lyon). Cabestan à écrevices, et cabestan à bras. L'un et l'autre accompagnés d'un modèle. 1741. p. 271-296, 3 pl.

T. V 
34. Dutour de Salvert, Etienne François (1711-1789). Discours sur l'aiman. (Essai sur l'aiman, où l'on explique son attraction avec le fer, la direction de l'aiguille aimantée vers le Nord, sa déclinaison, et son inclinaison.) 1744 . p. 49-114, 3 pl.

35. Euler, Johann Albrecht (1734-1800). Recherches sur l'arrimage des vaisseaux; et quelles bonnes qualités on en peut procurer à un vaisseau. 1761. p. $1-56$, et 3 pl. qui se trouvent dans le tome IX.

36. Euler, Leonhard (1707-1783). Dissertatio de igne, in qua ejus natura et proprietates explicantur. 1738. p.1-19.

37. - Inquisitio physica in causam fluxus ac refluxus maris. 1740. p. 235-350, $4 . \mathrm{pl}$.

T. IV

38. - De observatione inclinationis magneticae dissertatio. 1743. p. 63-96, 1 pl.

39. - Dissertatio de magnete. 1744. p. 1-47, 1 pl.

T. V

40. - Recherches sur les inégalités de Jupiter et de Saturne. 1752. p.1-84.

T. VII

41. - Examen des efforts qu'ont à soutenir toutes les parties d'un vaisseau dans le roulis et dans le tangage, ou recherches sur la diminution de ces mouvemens. 1759. p. 1-47, 2 pl.

42. - Théorie de la lune. 1770. p.1-94.

T. IX

43. - Nouvelles recherches sur le vrai mouvement de la lune. Où l'on détermine toutes les inégalités auxquelles il est assujetti. 1772. p.1-38.

T. IX

44. Fenel, Jean Basile Pascal (1695-1753). Recueil de différentes expériences, essais et raisonnemens sur la meilleure construction du cabestan; par rapport aux usages ausquels on l'applique dans un vaisseau. 1741. p. 217270,3 pl.

45. Groignard, Antoine (1727-1799). Mémoire composé à l'occasion du prix proposé : L'examen des efforts qu'ont à soutenir toutes les parties du vaisseau dans le roulis et dans le tangage. 1759. p. 1-51, $6 \mathrm{pl}$.

46. - Mémoire sur l'arrimage des vaisseaux. 1765. p. 1-36.

47. Lagrange, Joseph Louis de (1736-1813). Recherches sur la libration de la lune. 1764. p.1-50.

48. - Recherches sur les inégalités des satellites de Jupiter, causées par leur attraction mutuelle. 1766. p.1-162.

49. - Essai d'une nouvelle méthode pour résoudre le problème des trois corps. 1772. p.1-126.

T. IX

50. - Recherches sur la manière de former des tables des planètes, d'après les seules observations. 1772 . p. 513-616, 5 tables.

T. VIII

- La pagination se refère aux Mémoires de l'Académie de 1772, où la pièce a été publié d'abord.

51. - Recherches sur la théorie des perturbations que les comètes peuvent éprouver par l'action des planètes. 1772. p. 65-160. 
52. Lozeran du Fech [Fiesc], Louis Antoine (-1755). Discours sur la propagation du feu. 1738. p. 21-54.

53. Ludot, [? Jean Baptiste (1703-1771)]. Recherche de la meilleure construction du cabestan. 1741. p.121-193, $4 \mathrm{pl}$.

54. Poleni, Giovanni (1683-1761). Quanam potiori ratione, nullis habitis stellarum observationibus, navigantes metiri queant iter a navi confectum (De la meilleure manière de mesurer sur mer le chemin d'un vaisseau, indépendemment des observations astronomiques). 1733.40 p., 1 table, 2 pl.

55. - De praestabiliori figura qua anchora formari queant. - De artificio praestantiore anchoras ad ustrinam fabrefaciendi. - De meliore modo experiundi anchorarum vires, seu resistentiam. 1737. p. 85-159, 9 pl.

56. - De ergatae navalis praestabiliore, facilioreque usu, dissertatio. 1741. p. 89$119,5 \mathrm{pl}$.

57. Pontis d'Hurtis, le Cte de (-1745). Mémoire sur le cabestan. 1741. p.197-215, $2 \mathrm{pl}$.

58. Maclaurin, Colin (1698-1746). Démonstration des loix du choc des corps. 1724. 26 p., 1 pl.

59. - De causa physica fluxus et refluxus maris. 1740. p.193-234, 2 pl.

60. Massy. Quelles seroit la meilleure manière de conserver sur mer l'égalité du mouvement d'une pendule, soit par la construction de la machine, soit par sa suspension. $1720.32 \mathrm{p} ., 1 \mathrm{pl}$.

61. Mathon de la Cour, Jacques (1712-1770). Mémoire sur la manière la plus avantageuse de suppléer à l'action du vent sur les grands vaisseaux. 1753. p. $1-37,1 \mathrm{pl}$.

62. Mazière, Jean Simon (1679-1761). Les loix du choc des corps à ressort parfait, ou imparfait. 1726. 57 p., 1 pl.

63. - Traité des petits tourbillons de la matière subtile. 1726. 56 p.

T. I

64. Tresaguet, Octave (1674-1743). Mémoire sur la fabrique des ancres. 1737. p. 33-46, $11 \mathrm{pl}$.

65. Voltaire, François Marie Arouet de (1694-1778). Essai sur la nature du feu, et sur sa propagation. 1738. p. 169-217. [Anonyme]

66. Anonymes. Meditationes super problemate nautico, de implanatione malorum. 1727. 48 p., 2 pl.

67. - Dissertation sur la meilleure construction du cabestan. 1741. p. 29-87, $3 \mathrm{pl}$.

68. - De la meilleure manière de trouver l'heure en mer, par observation. 1745. p. $169-216,3 \mathrm{pl}$.

69. - Essai d'horolepse nautique. 1745. p. 217-456, 8 pl.

70. - Meditationes in quaestionem quibusnam observationibus mari ... verum temporis momentum ... determinari queat? 1747. p. 111-167, 2 pl. 
71. - Mémoire sur le programme pour le prix de 1747: La meilleure manière de trouver l'heure en mer, par observation. p. 457-526, 3 pl.

72. - Mémoire sur l'arrimage des vaisseaux. 1765. p.1-18.

T. IX

73. - Traité de l'arrimage des vaisseaux. 1765. p.1-95, 7 pl.

T. IX

\title{
Summary
}

The author gives a list of the prize-papers crowned by the Academy of Sciences at Paris from 1720 to 1772 . The essays are arranged according to the names of the authors. Far more than a third have been written by Swiss scientists.

\author{
Alvin Eugen Jaeggli \\ ETH-Bibliothek Zürich \\ Wissenschaftshistorische Sammlungen \\ 8093 Zürich
}

